\title{
CONSISTENT AND REGULARIZED MAGNIFICATION OF IMAGES
}

\author{
A. Bourquard, P. Thévenaz, K. Balać, and M. Unser
}

\author{
École polytechnique fédérale de Lausanne (EPFL), Biomedical Imaging Group \\ aurelien.bourquard@epfl.ch, philippe.thevenaz@epfl.ch, katarina.balac@epfl.ch, michael.unser@epfl.ch
}

\begin{abstract}
Because more output data must be created than is available from the input, magnification is an ill-posed problem. Traditional magnification relies on resampling an interpolation model at the appropriate rate; unfortunately, this simple solution is blind to the presence of the analog filter that was implicitly present when the samples of the function to be magnified were acquired. Consistent resampling has been introduced to take this into account, but it turns out that this solution is still under-constrained. In this paper, we propose regularization as a way to devise a deterministic magnification method that fully satisfies consistency constraints in the absence of noise, and at the same time that produces an output that best fulfills a wide class of criteria for regularity. Contrarily to many other methods, ours has been designed without ever leaving the continuous domain. We conduct experiments that show the benefit of our approach.
\end{abstract}

Index Terms-Generalized sampling, Consistency constraint, Projection operator, Ill-posed problem.

\section{INTRODUCTION}

The capacity to magnify digital images is often desirable, for example in the context of medical imaging or forensics. The challenging issue is the accuracy of the details at high magnification, which is especially important in the examples mentioned. All the available information from the original image has to be retained in its magnified version; moreover, the additional pixels introduced by the magnification process must remain as compatible as possible with the provided data.

Interpolation-based magnification methods are popular, where the output image $g$ with $p$ dimensions corresponds to the input image $f$ magnified by an integer factor $M$. By hypothesis, the input image belongs to the space spanned by the integer translates of an ana$\log$ generating (or synthesis) function $\varphi$, or, more precisely, so that $\forall \mathbf{x} \in \mathbb{R}^{p}: f(\mathbf{x})=\sum_{\mathbf{k} \in \mathbb{Z}^{p}} w[\mathbf{k}] \varphi(\mathbf{x}-\mathbf{k})$. The sequence of coefficients $w$ that are used as weights for the shifted synthesis functions $\varphi$ is obtained by a discrete convolution between the sampled image values $f[\mathbf{k}]$ at the original resolution, and a uniquely defined digital filter $q_{0}$, the latter being the identity for synthesis functions that satisfy $\varphi[\mathbf{k}]=\delta[\mathbf{k}]$, and a less-trivial filter otherwise [1]. Then, we simply have that $\forall \mathbf{x} \in \mathbb{R}^{p}: g(\mathbf{x})=f(\mathbf{x} / M)$. Since the synthesis function $\varphi$ is continuously defined, so is $g$, and this method allows for straightforward operations on the magnified image that include rotation, sub-pixel translation, gradient computation, and more. In practice, unfortunately, the samples must be acquired with the aid of some device with impulse response $\varphi_{0}$, which is discounted by the hypothesis that $f \in \operatorname{span}\{\varphi\}$. Moreover, the prefilter (or analysis function $\varphi_{0}$ ) exhibits non-idealities, which results in blurred, aliased, or even distorted magnified images.
Recently, a better method has been proposed in $[2,3]$ to magnify images while retaining the notion of a functional space. It uses the generalized-sampling theory, which takes simultaneously into account the analysis and synthesis functions $\varphi_{0}$ and $\varphi$, respectively. It takes advantage of a consistency constraint that states that the magnified signal $\tilde{f}$, after having been de-magnified in the continuous domain, filtered by $\varphi_{0}$, and subsequently re-sampled, must lead to the same measurements than the original signal $f$. This again requires a digital correction filter $q$, which plays a role similar to $q_{0}$. This concept has been applied to image magnification in [4]. Although magnification is an ill-posed problem, no regularization was used; therefore, no constraints were directly imposed on the magnified image. In addition, the method was not well-defined for an arbitrary number of dimensions.

Simply put, image magnification is an ill-posed problem because the outcome contains more pixels than the input can provide. Thus, arbitrary constraints are necessary to devise a deterministic magnification algorithm. One of these constraints goes by the name of consistency. But, as it turns out, this constraint is not sufficient to uniquely determine the magnified image, as several data configurations exist that lead to the same discrete set of filtered and re-sampled values. In this paper, we propose to take advantage of regularization to further constrain the algorithm. We show that a wide class of regularizers can be used and offer a generic solution to determine the discrete filter $q$ so that the continuously defined signal $\tilde{f}$ is at the same time regular, and consistent with the original continuously defined signal $f$. Our approach can be applied in an arbitrary number of dimensions.

\section{CONSISTENCY}

We present in Figure 1 the overall magnification scheme. The signals $f, f_{0}$, and $\tilde{f}$ are continuously defined, while the sequences $f_{1}$, $f_{2}$, and $c$ are discrete. The filter with impulse response $\varphi_{0}$ operates in the continuous domain, while the filter with impulse response $q$ operates in the discrete domain. Upsampling by $M$ must be understood as the insertion of $(M-1)$ zeroes between samples. A Dirac's comb portrays sampling, while the synthesis function $\varphi$ links the discrete world to the analog one. Overall, this results in $\forall \mathbf{k} \in \mathbb{Z}^{p}: f_{1}[\mathbf{k}]=\left.\left(\varphi_{0} * f\right)(\mathbf{x})\right|_{\mathbf{x}=\mathbf{k}}$. Finally, the chain of operations that transform the sequence of samples $f_{1}$ into the magnified output signal $\tilde{f}$ can be formalized as

$$
\forall \mathbf{x} \in \mathbb{R}^{p}: \tilde{f}(\mathbf{x})=\sum_{\mathbf{n} \in \mathbb{Z}^{p}}\left(\left\{f_{1}\right\}_{\uparrow_{M}} * q\right)[\mathbf{n}] \varphi(\mathbf{x}-\mathbf{n}) .
$$

To enforce consistency, we shall ask that a de-magnified version of $\tilde{f}$ result in the same set samples, after re-acquisition by $\varphi_{0}$ and re-sampling. We therefore write this recursive condition as 


$$
\begin{aligned}
\forall \boldsymbol{\omega} \in \mathbb{R}^{p}: J(\boldsymbol{\omega})= & \left|M^{2 p}-\sum_{\mathbf{m} \in[0 \ldots M-1]^{p} \backslash\{\mathbf{0}\}} A\left(\mathrm{e}^{\mathrm{j}\left(\boldsymbol{\omega}+\frac{2 \pi \mathbf{m}}{M}\right)}\right) Q\left(\mathrm{e}^{\mathrm{j}\left(\boldsymbol{\omega}+\frac{2 \pi \boldsymbol{m}}{M}\right)}\right)\right|^{2} \mathrm{~L}_{\hat{\varphi}}(\boldsymbol{\omega}) \\
& +\left|A\left(\mathrm{e}^{\mathrm{j} \boldsymbol{\omega}}\right)\right|^{2} \sum_{\mathbf{m} \in[0 \ldots M-1]^{p} \backslash\{\mathbf{0}\}}\left|Q\left(\mathrm{e}^{\mathrm{j}\left(\boldsymbol{\omega}+\frac{2 \pi \mathrm{m}}{M}\right)}\right)\right|^{2} \mathrm{~L}_{\hat{\varphi}}\left(\boldsymbol{\omega}+\frac{2 \pi \mathbf{m}}{M}\right) .
\end{aligned}
$$

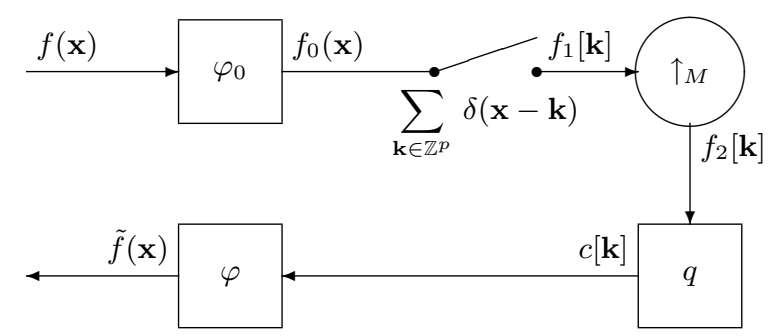

Fig. 1. Magnification system. The continuously defined function $f$ is submitted to an acquisition device $\varphi_{0}$ before being sampled at unit intervals. The resulting discrete sequence of samples $f_{1}$ is then upscaled by $M$ and digitally filtered by $q$. The magnified and continuously defined output signal is $\tilde{f}$.

$$
\begin{aligned}
& \forall \mathbf{k} \in \mathbb{Z}^{p}: f_{1}[\mathbf{k}]= \\
& \left.\quad\left(\varphi_{0} * \sum_{\mathbf{n} \in \mathbb{Z}^{p}}\left(\left\{f_{1}\right\}_{\uparrow_{M}} * q\right)[\mathbf{n}] \varphi(M \cdot-\mathbf{n})\right)(\mathbf{x})\right|_{\mathbf{x}=\mathbf{k}},
\end{aligned}
$$

where we identify a discrete convolution between sequences $a$ and $b$ by putting its argument into brackets, like in $(a * b)[\cdot]$, and where we identify a continous convolution between signals $a$ and $b$ by putting its argument into parentheses, like in $(a * b)(\cdot)$.

After some calculations, we conclude that the consistency condition can be satisfied for any sequence $f_{1}$, provided the filter with discrete impulse response $q$ satisfies the condition

$$
\forall \boldsymbol{\omega} \in \mathbb{R}^{p}: M^{2 p}=\sum_{\mathbf{m} \in[0 \ldots M-1]^{p}} A\left(\mathrm{e}^{\mathrm{j} \frac{2 \pi \mathbf{m}+\boldsymbol{\omega}}{M}}\right) Q\left(\mathrm{e}^{\mathrm{j} \frac{2 \pi \mathbf{m}+\boldsymbol{\omega}}{M}}\right),
$$

where $Q(\mathbf{z})=\sum_{\mathbf{k} \in \mathbb{Z}^{p}} q[\mathbf{k}] \mathbf{z}^{-\mathbf{k}}$ is the $p$-dimensional $\mathbf{z}$-transform of the sequence $q$, and where $A$ is the $\mathbf{z}$-transform of the sampled continuous cross-correlation between the magnified analysis function and the synthesis function given by

$$
\forall \mathbf{k} \in \mathbb{Z}^{p}: a[\mathbf{k}]=\left.\left(\varphi_{0}\left(\frac{\cdot}{M}\right) * \varphi\right)(\mathbf{x})\right|_{\mathbf{x}=\mathbf{k}} .
$$

\section{REGULARIZATION}

Because the consistency constrain $M^{2 p}=\sum A Q$ is a single linear equation in terms of as many as $M^{p}$ unknowns, the null-space of the system described up to this point is far from being empty. In other words, one can find many functions $\eta$ such that $\tilde{\eta}=0$. This means that the functions $(f+\eta)$ are consistent with $f$ in the sense of Section 2, and so there are many consistent solutions to the magnification problem. To reclaim unicity, among all these solutions we are going to pick the most regular one, in the sense defined below.
To measure the regularity of a function, we first apply an operator $\mathrm{L}$ that can take any form which can be described as a convolution by a filter with impulse response $h$, so that $\forall \mathbf{x} \in \mathbb{R}^{p}: \operatorname{L}\{f\}(\mathbf{x})=$ $(h * f)(\mathbf{x})$. Typical choices for L involve gradients and Laplacians, in which case $h$ relates to the derivatives of Dirac's $\delta$, in the sense of distributions. Then, we take the $L_{2}$-norm of the outcome. After the appropriate calculations, we conclude in our case that the roughness - the opposite of the regularity — of the magnified signal must be measured as

$$
\|\mathrm{L}\{\tilde{f}\}\|^{2}=\frac{1}{(2 \pi)^{p}} \int_{\left[0, \frac{2 \pi}{M}\right]^{p}}\left|\frac{F_{1}\left(\mathrm{e}^{\mathrm{j} M \boldsymbol{\omega}}\right)}{A\left(\mathrm{e}^{\mathrm{j} \boldsymbol{\omega}}\right)}\right|^{2} J(\boldsymbol{\omega}) \mathrm{d}^{p} \omega,
$$

where $F_{1}$ is the $\mathbf{z}$-transform of the sequence $f_{1}$, where the auxiliary function $J$ is defined as in (1), and where the function $\mathrm{L}_{\hat{\varphi}}$ depends on the synthesis function and on the regularization filter, but neither on the signal nor on the analysis filter. More precisely, $\mathrm{L}_{\hat{\varphi}}$ is defined as

$$
\forall \boldsymbol{\omega} \in \mathbb{R}^{p}: \mathrm{L}_{\hat{\varphi}}(\boldsymbol{\omega})=\sum_{\mathbf{m} \in \mathbb{Z}^{p}}|\hat{h}(\boldsymbol{\omega}+2 \pi \mathbf{m}) \hat{\varphi}(\boldsymbol{\omega}+2 \pi \mathbf{m})|^{2},
$$

where $\hat{h}(\boldsymbol{\omega})=\int_{-\infty}^{\infty} h(\mathbf{x}) \mathrm{e}^{-\mathrm{j}\langle\boldsymbol{\omega}, \mathbf{x}\rangle} \mathrm{d}^{p} x$, and $\hat{\varphi}$, are the Fourier transforms of the regularization filter and of the synthesis function, respectively.

By hypothesis, the functions $\varphi, \varphi_{0}$, and $h$ are given. Therefore, only $q$ can be tuned to minimize our measure of roughness. As the two terms $\left|F_{1} / A\right|^{2}$ and $J$ in (2) are nonnegative, and since only $J$ depends on $q$, we can concentrate on minimizing $J$, independently for each pulsation $\boldsymbol{\omega}$. This has the advantage of yielding a simple algorithm with the same filter $q$ for all signals $f$. One distinguishing feature of our proposed method is that this optimization process can be conducted through analysis in the continuous domain. After several pages of tedious calculations that we once more skip in the interest of space, we determine the $\mathbf{z}$-transform of the optimal compensation filter to be given by

$$
\begin{aligned}
& \forall \boldsymbol{\omega} \in \mathbb{R}^{p}: Q\left(\mathrm{e}^{\mathrm{j} \boldsymbol{\omega}}\right)= \\
& M^{2 p} A^{*}\left(\mathrm{e}^{\mathrm{j} \boldsymbol{\omega}}\right) \\
& \mathrm{L}_{\hat{\varphi}}(\boldsymbol{\omega}) \sum_{\mathbf{m} \in[0 \ldots M-1]^{p}}\left|A\left(\mathrm{e}^{\mathrm{j}\left(\boldsymbol{\omega}+\frac{2 \pi \mathbf{m}}{M}\right)}\right)\right|^{2} \frac{1}{\mathrm{~L}_{\hat{\varphi}\left(\boldsymbol{\omega}+\frac{2 \pi \mathbf{m}}{M}\right)}}
\end{aligned}
$$

\subsection{Implementation}

Obtaining explicit expressions for $q$ often proves to be difficult. For those many difficult cases, we advocate the use of discrete Fourier transforms as a practical way to implement the system described in Figure 1. The most delicate issue is the computation of the term $\mathrm{L}_{\hat{\varphi}}(\boldsymbol{\omega}) / \mathrm{L}_{\hat{\varphi}}\left(\boldsymbol{\omega}+\frac{2 \pi \mathbf{m}}{M}\right)$ found in $Q$. How to best approximate this quantity depends on the interplay between the decays in frequency of $\hat{h}$ and $\hat{\varphi}$. 


\section{EXPERIMENTS}

In this section, our purpose is to assess the visual performance of our algorithm, as compared to traditional magnification by interpolation. In both cases, the type and maximum extent to which images are detailed is governed by the synthesis function $\varphi$ of Figure 1 . To ensure a fair comparison, we have imposed the same $\varphi$ for traditional interpolation and for our proposed method; presently, we have set $\forall \mathbf{x} \in \mathbb{R}^{p}: \varphi(\mathbf{x})=\prod_{i=1}^{p} \max \left(0,1-\left|x_{i}\right|\right)$, which is generally called linear interpolation. Then, one difference between our method and the traditional one is the application of the filter with impulse response $q$. This filter takes into account-and compensates forthe existence of $\varphi_{0}$, which traditional interpolation does not.

The specification of the filter $q$ requires that some regularizer has been chosen a priori. In the present case, we have opted for the Laplacian-like form $\forall \boldsymbol{\omega} \in \mathbb{R}^{p}: \hat{h}(\boldsymbol{\omega})=\left(\sum_{i=1}^{p} \omega_{i}^{2}\right)^{8}$. This regularizer penalizes abrupt transitions in the image, which tends to suppress high-frequency noise. Meanwhile, piecewise constant areas or gentle transitions such as shadows are not penalized, which tends to maintain a natural aspect to the resulting magnified images. Once more, let us insist that the role of regularization is only to pick one consistent solution out of many; it is not to be understood as a tradeoff factor.

Finally, the analysis function has been assumed to correspond to $\forall \mathbf{x} \in \mathbb{R}^{p}: \varphi_{0}(\mathbf{x})=\operatorname{rect}(\mathbf{x})$. This is the model of an idealized CCD sensor, where the sensing area of each pixel would be a small square, and where all these squares would be contiguous to cover the whole sensor. We have set the up-sampling factor to be $M=4$.

\subsection{Barbara}

In our first visual experiment, the input image $f$ is the fragment the classical Barbara image that we show in Figure 2. It depicts the long and tight stripes of Barbara's trousers, which have a strong grayscale contrast. We show in Figure 3 a version of $f$ magnified by traditional linear interpolation, while we present in Figure 4 the outcome of our algorithm. We observe that consistent and regularized magnification results in an image that is sharper than that resulting from standard magnification by interpolation. Edge details are more accurate and better distinguishable, especially when the stripes get tighter on the image. We have attempted to enhance Figure 3 by post-processing with sharpening operations (results not shown), but obtained only poorer results, with a significant amount of added noise.

The improvement over edge details can be explained in part by the high-order Laplacian used for regularization, which allows for an accurate magnification of oscillating patterns. Indeed, when we observe plot profiles from the image of Figure 4, only very smooth, almost sine-like oscillating patterns appear, contrarily to those found in Figure 3. We therefore claim that such a regularization operator, along with the fact that the analysis function has been taken into account, allows for the efficient recovery of those oscillating patterns during magnification.

\subsection{MRI}

In our second experiment, the input image $f$ is the magnetic resonance image (MRI) that we show in Figure 5. Keeping the same order as in Section 4.1 for Figures 5-7, we observe that our method produces more accurate textures, and transition patterns with less perceptible staircases.

In case of regularized magnification, reduced blur is due to the fact that we resist assuming the analysis function to be ideal; instead,

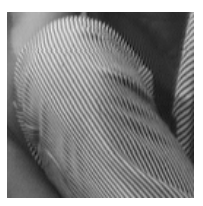

Fig. 2. Detail of the Barbara image, at the original size $128 \times 128$

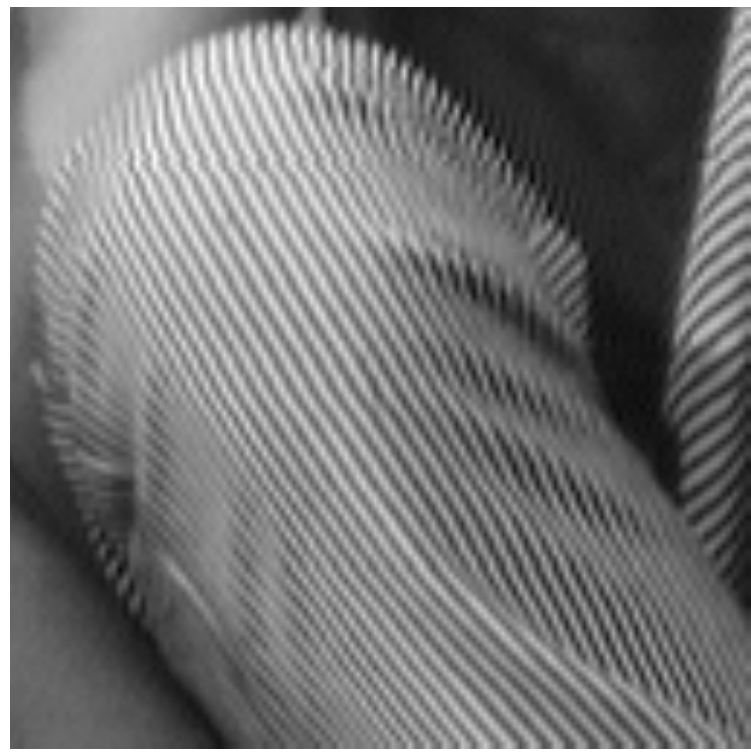

Fig. 3. Detail of the Barbara image after magnification by standard interpolation, with size $512 \times 512$.

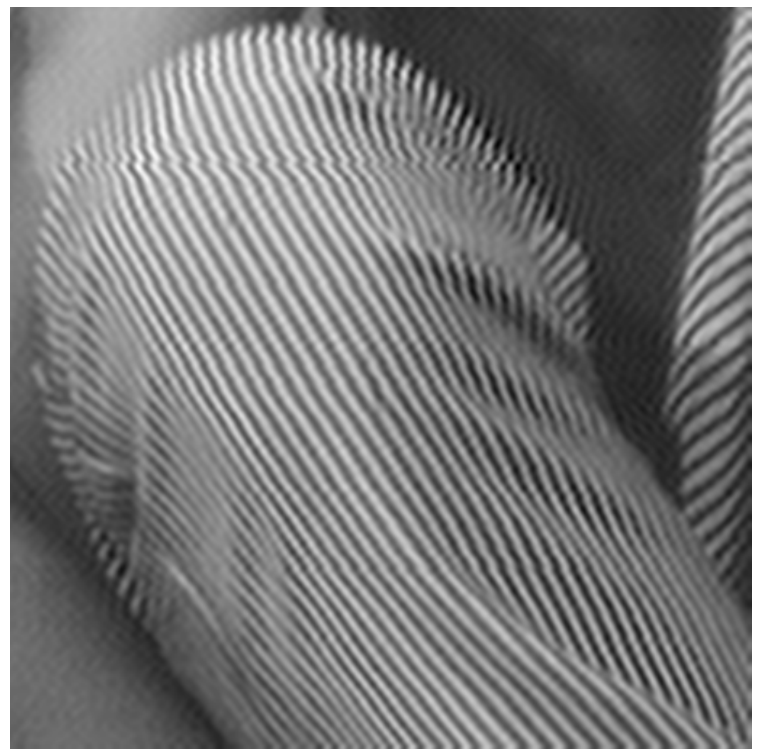

Fig. 4. Detail of the Barbara image after our consistent and regularized magnification, with size $512 \times 512$. 
we assume it is a rect low-pass filter, which is realistic when considering most image acquisition devices. Thus, the correction filter $q$ restores some attenuated details. Moreover, since the linear operator chosen for regularization is the high-order Laplacian $\Delta^{8}$, which is rotation-invariant, all high-frequency elements are heavily penalized in all directions. This explains the high continuity of the transition patterns in the magnified images, and the absence of staircase effects which are predominantly found at high-frequencies.

\section{CONCLUSION}

We have developed a high-quality magnification method that combines consistency and regularization constraints. We deal with the regularization term in the continuous domain, yet the solution takes the form of a digital filter. Our method is linear, in the sense that magnifying the sum of two images or summing two magnified images produces the same outcome. It shows interesting results when compared to magnification using standard interpolation. Texture details as well as transitions are more accurate, and blur is reduced. In addition, our method does not depend on the number of dimensions.

\section{ACKNOWLEDGMENTS}

This work was supported by the Center for Biomedical Imaging (CIBM) of the Geneva-Lausanne universities and the EPFL, as well as by the Hasler, Leenaards, and Louis-Jeantet foundations.

\section{REFERENCES}

[1] M. Unser and A. Aldroubi, "A General Sampling Theory for Nonideal Acquisition Devices," IEEE Transactions on Signal Processing, vol. 42, no. 11, pp. 2915-2925, November 1994.

[2] L. Condat, Méthodes d'approximation pour la reconstruction de signaux et le redimensionnement d'images, 249 p., Institut National Polytechnique de Grenoble, September 18, 2006.

[3] T. Yokokawa, M. Kamada, Y. Ohtaki, and T. Yonekura, "Estimation of Color Images by Box Splines from Their Observation through Honeycomb Color Filter," IEICE Transactions on Fundamentals of Electronics, Communications and Computer Sciences, vol. E89-A, no. 12, pp. 3678-3684, December 2006.

[4] A. Hirabayashi and M. Unser, "Consistent Sampling and Signal Recovery," IEEE Transactions on Signal Processing, vol. 55, no. 8, pp. 4104-4115, August 2007.

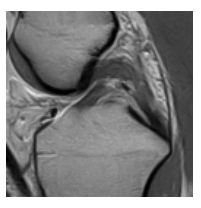

Fig. 5. MRI image, at the original size $128 \times 128$.

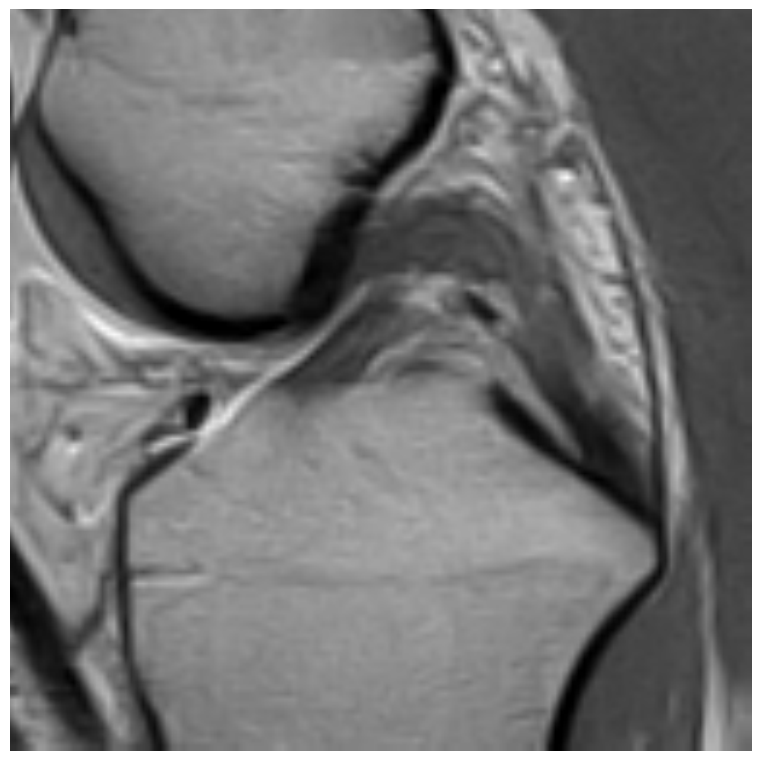

Fig. 6. MRI image magnified by standard interpolation, with size $512 \times 512$.

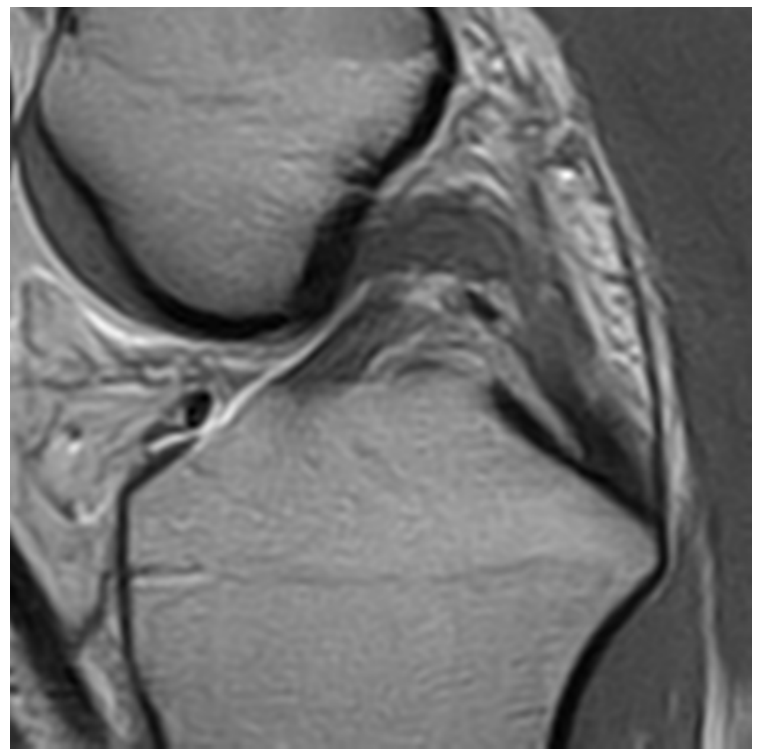

Fig. 7. MRI image after our consistent and regularized magnification, with size $512 \times 512$. 